\title{
Types of urethral stricture and their recurrence rates post urological treatments
}

\author{
Wedyan Salem Basaif ${ }^{1 *}$, Husam Hamad Alamri², Hind Waleed Mousa ${ }^{3}$, \\ Raghad Abdulelah Alsayed ${ }^{4}$, Abdullah Mohammed Almohammadi ${ }^{2}$, \\ Waleed Hamed Altulayqi ${ }^{5}$, Abdulrahman Mohammed Albejawi ${ }^{4}$, \\ Ammar Abdullah Allam², Suliman Abdulrahman Alnamlah ${ }^{5}$, \\ Mohammed Siraj Alghamdi ${ }^{1}$, Sarah Mohammed Mahrous ${ }^{2}$
}

\author{
${ }^{1}$ Department of Urology, King Fahad General Hospital, Jeddah, Saudi Arabia \\ ${ }^{2}$ Department of Urology, King Fahad General Hospital, Medina, Saudi Arabia \\ ${ }^{3}$ College of Medicine, Medical University of Warsaw, Warsaw, Poland \\ ${ }^{4}$ Department of Urology, Ohud Hospital, Medina, Saudi Arabia \\ ${ }^{5}$ Department of Urology, Prince Mohammed Bin Abdulaziz Hospital, Riyadh, Saudi Arabia
}

Received: 25 May 2021

Accepted: 09 June 2021

\section{*Correspondence:}

Dr. Wedyan Salem Basaif,

E-mail: wedyanbasaif@gmail.com

Copyright: (C) the author(s), publisher and licensee Medip Academy. This is an open-access article distributed under the terms of the Creative Commons Attribution Non-Commercial License, which permits unrestricted non-commercial use, distribution, and reproduction in any medium, provided the original work is properly cited.

\begin{abstract}
Urethral strictures can significantly impact the quality of life for patients because it can be associated with significant complications such as fistulas, bladder calculi, infections and sepsis. Additionally, it might even lead to renal failure. The worldwide prevalence of urethral strictures is high, with an estimated rate of 229-627 patients per 100,000 population. In this literature review, the aim was to discuss the types and etiology of urethral strictures and the recurrence rates following the different management modalities. Studies that were included in this review were published between January 2005 until May 2021. The results support the current evidence that the idiopathic and iatrogenic bulbar strictures are the most common types while penile strictures, the iatrogenic and inflammatory are the most common causes. Recurrence rates are reported after management with almost all of the current management modalities, indicating the need for better interventions to enhance the outcomes and alleviate the quality of care. The recurrence rate of strictures after treatment with internal urethrotomy and direct vision internal urethrotomy by three years is $65 \%$. Other studies reported that the rate of recurrence was estimated to be around 14 after 6 months from internal urethrotomy and up to $27 \%$ after 12 months. The rate of complications and recurrence following treatment with anastomotic urethroplasty was estimated to be less than $5 \%$. Detailed information and discussion were provided in the study manuscript.
\end{abstract}

Keywords: Urology, Urethrotomy, Strictures, Etiology, Epidemiology

\section{INTRODUCTION}

Estimates from the United States show that a total of 5,000 inpatients hospital visits are recorded per year. In addition, around 1.5 million office visits that are attributable to male urethral strictures. ${ }^{1}$ The latter can significantly impact the quality of life for patients because it can be associated with significant complications such as fistulas, bladder calculi, infections, sepsis. Additionally, it might even lead to renal failure. A previous investigation by Mundy et al estimated the rates of complications as investigated from previous 
investigations that were conducted in patients suffering from untreated urethral strictures. ${ }^{1}$ In the United States, estimates show that the incidence of urethral strictures is $0.9 \%$ per year, while in the United Kingdom, it was previously estimated that the prevalence is 40 per 100,000 population in individuals that are 65 years old and increases to 100 patients in older individuals., ${ }^{1,2}$ The worldwide prevalence of urethral strictures is even higher with an estimated rate of 229-627 patients per 100,000 population. ${ }^{3}$ In this literature review, the aim was to discuss the types of urethral strictures and its etiology along with the recurrence rates following the different management modalities.

The complications of untreated urethral strictures are thick-walled trabeculated bladder ( $85 \%$ incidence), acute retention (60\% incidence), prostatitis (50\% incidence), epididymo-orchitis (25\% incidence), hydronephrosis (20\% incidence), periurethral abscess (15\% incidence) and bladder or urethral stones (10\% incidence).

\section{METHODS}

A systematic search was conducted to identify relevant studies in the following databases: PubMed, Medline, Web of Science, Embase, Google Scholar and Scopus. The following search terms were used (urethral stricture) and (recurrence) and (post or after) and (urological treatment or urological outcome). The reference lists were manually searched to identify additional relevant studies meeting inclusion criteria. No restrictions were applied.

\section{DISCUSSION}

\section{Types of urethral strictures}

The most common classification of urethral strictures is by etiology. Two main types of urethral strictures have been reported including the bulbar and penile strictures. The main causes of urethral strictures are idiopathic, iatrogenic, inflammatory and traumatic with estimated prevalence rates of $40 \%, 35 \%, 10 \%$, and $15 \%$ for each, respectively. ${ }^{1}$ Overall, these four types represent the most common etiologies for all urethral strictures and estimates also show that the iatrogenic and idiopathic types represent the most common types for all urethral strictures and account for around one third each. Traumatic causes were also reported to follow these two types in order followed by the inflammatory types which represent a total of $19 \%$ and $15 \%$, respectively among the various types of urethral strictures. ${ }^{4}$ Regarding the development and pathology of idiopathic types, previous studies have demonstrated that the underlying mechanism behind the development of these types is the potential presence of previous penile traumas that repeatedly occurred and finally led to the development of a penile stricture. ${ }^{5}$ On the other hand, iatrogenic urethral strictures have been reported to be caused by five main causes. Previous estimates showed that trans-urethral resections (TURs) represent the most common cause of the iatrogenic urethral strictures, accounting for $41 \% .^{4}$ The mechanism behind the development of the stricture is mainly explained by the stretching of the urethra and inducing significant damage to the underlying epithelium due to the frequent passing of the instruments up and down the urethra. ${ }^{1}$ Following TURs, prolonged catheterization also constitutes a common etiology for developing iatrogenic urethral strictures with an estimated frequency rate of $36 \% .^{4}$ The main mechanism by which urethral catherization induces strictures is by induction of severe pressure to the underlying urethra leading to a significant injury and necrosis to the underlying epithelium and contributing to the development of urethral strictures. However, many advances have been made to reduce the prevalence and incidence of urethral strictures following catheter installation. ${ }^{6}$ Many substances have been proposed to reduce the injury and pressure on the urethra by using silicon in synthesizing the catheter rather than latex. However, reports also show the persistence and development of strictures following the intermittent application of the catheterization techniques, which will eventually lead to the development of urethral strictures following the installation over a prolonged period. Iatrogenic strictures following cystoscopy administration were also reported in the literature to constitute a total of $12.7 \%$ of iatrogenic strictures, owing to significant epithelial injury. ${ }^{4}$ Besides, around $6.3 \%$ of the reported iatrogenic strictures were also reported to be caused by hypospadias repair. ${ }^{4}$ In this context, it was previously estimated that performing hypospadias repair procedures during childhood significantly increases the risk of developing strictures by $10 \%$ later on. ${ }^{6}$ Among the reported causes of iatrogenic strictures, prostatectomy has been reported to attribute to $3.2 \%$ of the cases. ${ }^{4}$ Moreover, many male procedures and their management approaches have been associated with the development of iatrogenic strictures as the approaches that are associated with prostate cancer, including chemotherapy, prostatectomy and radiotherapy, which has been previously reported to account for $8.4 \%$ of the causes of urethral strictures that can also occur in the bulbar or posterior urethra. ${ }^{7}$ However, the exact cause and mechanism behind the development of this complication secondary to these procedures are still poorly understood and some authors attribute it to the potential injury to the urethra by the used instruments during any of these approaches or might be due to bladder stenosis secondary to performing radical prostatectomy. ${ }^{8}$ The development of strictures secondary to inflammation might be attributable to a pre-existing infection, which can significantly lead to narrowing of the urethral lumen and damage to the underlying epithelium. This was most commonly reported following the presence of recurrent gonococcal urethritis. Nevertheless, previous estimates showed that these types are no longer common within the population residing in developed countries unlike those within developing countries, which might be attributable to the healthcare advances and population education. ${ }^{2}$ Despite being poorly understood, previous studies have also expressed a potential link between certain infections as tuberculosis, 
chlamydia and schistosomiasis and the development of infectious post-inflammatory urethral strictures. ${ }^{6}$ Many other inflammation-causing infections as recurrent urinary tract infections by Escherichia coli and Lichen sclerosis were also reported among studies in the literature as potential causes of developing urethral strictures. ${ }^{8,9}$ Although the exact mechanism is still vague, genetic predisposition might play a role. Inflammatory urethral strictures can be differentiated from other strictures by other etiologies by being observed in the anterior part of the urethra only and also by being more longer than other types of strictures., ${ }^{2,10}$

\section{Treatment and post-treatment recurrence}

Although many approaches have been previously reported for the different types of urethral strictures, these disorders can be complicated by recurrence in the following post-treatment period. It should be noted that the management of urethral strictures is mainly based on the presence of complications. For patients with no complications, the treatment is only done to relieve the underlying symptoms. ${ }^{1,11}$ On the other hand, whenever complications were observed as the presence of recurrent infections and acute retention of urine, the management would be mainly directed to relieve the complications as a priority. Overall, the treatment of urethral strictures can be mainly divided into open surgical (by resection of the stricture urethroplasty, anastomosis and perineal urethrostomy) and transurethral (by internal urethrotomy and dilatation). Previous studies have reported that complications can occur with the two types of management and recurrence of strictures was also reported with the two management modalities, especially with previously recurrent strictures and the long ones. ${ }^{12}$ Evidence shows that urethral dilatation has been commonly used as the standard first-line management modality of urethral strictures. The mechanism by which urethral dilatation acts is by dilatation of the stricture by using sounds and boogies which increase the size of the urethra at the site of the stricture. Previous studies showed that the outcomes following the application of internal urethral dilatation were statistically significant from the outcomes obtained with using internal direct vision urethrotomy. ${ }^{1,11,13}$ Moreover, it was previously reported that the recurrence rate of strictures following internal urethral dilatation occurs in $65 \%$ of the cases following intervention by three years. ${ }^{14}$ Besides, other disadvantages of the procedure might include discomfort, pain and bleeding since the procedure is usually conducted under local anesthesia. Accordingly, using a balloon dilatation was previously suggested to decrease the severity of trauma. Moreover, it has been suggested that approaching dilatation by this modality can be associated with reduced recurrence rates. Besides, it was also reported that the procedure can be effectively used as a clean intermittent self-catheterization after the administration of internal visual urethrotomy. ${ }^{14} \mathrm{~A}$ previous investigation by Aldemir et al reported that the prevalence rate of urethral strictures following internal urethrotomy was $13.6 \%$ at 6 months and $27.3 \%$ at 12 months. ${ }^{15}$ Moreover, the authors reported that no significant association was noticed with the risk of stricture recurrence, stricture length or location, bladder capacity, the wall thickness of the bladder or the estimated length of the widest part of the affected urethra.

Another treatment modality that has been reported in the literature is internal direct vision urethrotomy, which has been previously recommended to be applied as the first line of choice for the management of short bulbar strictures because it was reported to be highly efficacious in such cases with estimated high success rates compared to other modalities. ${ }^{16}$ As previously mentioned, the recurrence rate of strictures after treatment with the modality by three years is $65 \% .{ }^{14}$ Moreover, a rate of $6.5 \%$ for complications was estimated following this modality and erectile dysfunction accounts for a total of $5 \%$ of the total complications followed by urinary incontinence, extravasation, urinary tract infections and hematuria accounting for prevalence rates of $4 \%, 3 \%, 2 \%$ and $2 \%$, respectively. If recurrence occurs following direct vision internal urethrotomy, evidence about reperforming the modality is controversial. Some studies suggest that it should be performed after the first time of recurrence while other authors suggest that urethroplasty should be performed whenever recurrence was observed following the first treatment. ${ }^{13,17}$ This was furtherly explained by the potential elongation of the stricture following the first procedure as a result of the excised distal and proximal ends. ${ }^{12}$ It should be noted that prophylactic antibiotics should be prescribed to prevent any potential infections that might complicate the case. ${ }^{1}$ End-to-end-anastomosis and urethral resection is commonly used to describe anastomotic urethroplasty. ${ }^{2}$ The procedure is commonly recommended for short strictures within the bulbar urethra that occur secondary to straddle injury and is usually favorable to patients for whom this will be the first interventional procedure for managing strictures. ${ }^{18}$ On the other hand, the procedure should not be performed with longer penile strictures due to the potential to cause ventral penile curvatures to the affected patients. The procedure has been reported with many advantages, and the most important ones include the high success rate being $90 \%$ and the low complications rate, including recurrence of strictures, which is estimated to attribute to $5 \%$ only. ${ }^{11,1718}$ Other management procedures with fewer complications and recurrence rates were also reported but are more complex than the aforementioned procedures. For too long bulbar strictures, substantial urethroplasty was previously reported in the literature as the most suitable procedure. ${ }^{11,19}$ The procedure is flexible and can be used for managing many complex cases and cases where other treatment modalities failed to achieve favorable outcomes. ${ }^{13,20}$ Another modality is perineal urethrostomy which has been previously reported to be indicated for repeatedly recurrent cases and in cases where significant comorbidities were present. Significant satisfaction with 
the procedure by the corresponding patients was previously reported. ${ }^{12}$

\section{CONCLUSION}

In this systematic review, it has been discussed the different types and of urethral strictures and its etiologies along with the recurrence rates that are associated with the different management modalities. The results support the current evidence that the idiopathic and iatrogenic bulbar strictures are the most common types while penile strictures, the iatrogenic and inflammatory causes are the most common. Recurrence rates are reported after management with almost all of the current management modalities, indicating the need for better interventions to enhance the outcomes and alleviate the quality of care.

\section{Funding: No funding sources}

Conflict of interest: None declared

Ethical approval: Not required

\section{REFERENCES}

1. Mundy AR, Andrich DE. Urethral strictures. BJU Int. 2011;107(1):6-26.

2. Smith TG. Current management of urethral stricture disease. Ind J Urol. 2016;32(1):27-33.

3. Liaw A, Rickborn L, McClung C. Incidence of urethral stricture in patients with adult acquired buried penis. Adv Urol. 2017;2017:7056173.

4. Fenton AS, Morey AF, Aviles R, Garcia CR. Anterior urethral strictures: etiology and characteristics. Urology. 2005;65(6):1055-8.

5. Viers BR, Pagliara TJ, Rew CA, Cooley LF, Shiang CY, Scott JM, et al. Characteristics of idiopathic urethral strictures: a link to remote perineal trauma? Urology. 2017;110:228-33.

6. Latini JM, McAninch JW, Brandes SB, Chung JY, Rosenstein D. SIU/ICUD consultation on urethral strictures: epidemiology, etiology, anatomy, and nomenclature of urethral stenoses, strictures, and pelvic fracture urethral disruption injuries. Urology. 2014;83(3):1-7.

7. Anger JT, Santucci R, Grossberg AL, Saigal CS. The morbidity of urethral stricture disease among male medicare beneficiaries. BMC Urol. 2010;10:3.

8. Mathur R, Aggarwal G, Satsangi B, Khan F, Odiya $\mathrm{S}$. Comprehensive analysis of etiology on the prognosis of urethral strictures. Clinical Urology. 2011;37(3):362-9.
9. Kirtschig G. Lichen sclerosus-presentation, diagnosis and management. Dtsch Arztebl Int. 2016;113(19):337-43.

10. Levy A, Browne B, Fredrick A, Stensland K, Bennett J, Sullivan T, et al. Insights into the pathophysiology of urethral stricture disease due to Lichen sclerosus: comparison of pathological markers in Lichen Sclerosus induced strictures vs nonlichen sclerosus induced strictures. J Urol. 2019;201(6):1158-63.

11. Mundy AR. Management of urethral strictures. Postgrad Med J. 2006;82(970):489-93.

12. Tritschler S, Roosen A, Füllhase C, Stief CG, Rübben H. Urethral stricture: etiology, investigation and treatments. Dtsch Arztebl Int. 2013;110(13):220-6.

13. Cheng L, Li S, Wang Z, Huang B, Lin J. A brief review on anterior urethral strictures. Asian J Urol. 2018;5(2):88-93.

14. Vyas JB, Ganpule AP, Muthu V, Sabnis RB, Desai MR. Balloon dilatation for male urethral strictures revisited. Urology Ann. 2013;5(4):245-8.

15. Aldemir M, Isık E, Okulu E, Ener K, Kayıil Ö. Urethral and bladder changes and stricture recurrence rates following internal urethrotomy for short urethral strictures. Uro Today Int J. 2011;4:27.

16. Hampson LA, McAninch JW, Breyer BN. Male urethral strictures and their management. Nature Rev Urol. 2014;11(1):43-50.

17. Fuehner C, Dahlem R, Fisch M, Vetterlein MW. Update on managing anterior urethral strictures. Ind J Urol. 2019;35(2):94-100.

18. Mundy AR. Anastomotic urethroplasty. BJU Int. 2005;96(6):921-44.

19. Horiguchi A. Substitution urethroplasty using oral mucosa graft for male anterior urethral stricture disease: current topics and reviews. Int $\mathbf{J}$ Urol. 2017;24(7):493-503.

20. Hoy NY, Chapman DW, Rourke KF. Better defining the optimal management of penile urethral strictures: a retrospective comparison of single-stage vs. two-stage urethroplasty. Canadian Urol Assoc J. 2019;13(12):414-8.

Cite this article as: Basaif WS, Alamri HH, Mousa HW, Alsayed RA, Almohammadi AM, Altulayqi WH, et al. Types of urethral stricture and their recurrence rates post urological treatments. Int J Community Med Public Health 2021;8:3634-7. 